\title{
The role of polymorphisms in glutathione- related genes in asbestos-related diseases
}

\author{
Alenka Franko1,2, Katja Goricar³, Metoda Dodic Fikfak¹,2, Viljem Kovac ${ }^{2,4}$, Vita Dolzan³ \\ ${ }^{1}$ Clinical Institute of Occupational Medicine, University Medical Centre Ljubljana, Ljubljana, Slovenia \\ ${ }^{2}$ Faculty of Medicine, University of Ljubljana, Ljubljana, Slovenia \\ ${ }^{3}$ Pharmacogenetics Laboratory, Institute of Biochemistry and Molecular Genetics, Faculty of Medicine, University of \\ Ljubljana, Ljubljana, Slovenia \\ ${ }^{4}$ Institute of Oncology Ljubljana, Ljubljana, Slovenia
}

Radiol Oncol 2021; 55(2): 179-186.

Received 9 November 2020

Accepted 2 December 2020

Correspondence to: Prof. Vita Dolžan, M.D., Ph.D., Pharmacogenetics Laboratory, Institute of Biochemistry and Molecular Genetics, Faculty of Medicine, University of Ljubljana, Vrazov trg 2, SI-1000 Ljubljana, Slovenia; Phone +386 15437670 ; GSM: +386 51 625 455; Fax: + 386154376 41; E-mail: vita.dolzan@mf.uni-lj.si

Disclosure: No potential conflicts of interest were disclosed.

\begin{abstract}
Background. The study investigated the influence of GCLC, GCLM, GSTMl, GSTI and GSTP1 polymorphisms, as well as the influence of interactions between polymorphism and interactions between polymorphisms and asbestos exposure, on the risk of developing pleural plaques, asbestosis and malignant mesothelioma (MM).

Subjects and methods. The cross sectional study included 940 asbestos-exposed subjects, among them 390 subjects with pleural plaques, 147 subjects with asbestosis, 225 subjects with MM and 178 subjects with no asbestos-related disease. GCLC rs17883901, GCLM rs41303970, GSTM1 null, GST1 null, GSTP1 rs1695 and GSTP1 rs1 138272 genotypes were determined using PCR based methods. In statistical analysis, logistic regression was used.

Results. GSTI null genotype was associated with the decreased risk for pleural plaques $(\mathrm{OR}=0.63 ; 95 \% \mathrm{Cl}=0.40$ $0.98 ; \mathrm{p}=0.026)$ and asbestosis $(\mathrm{OR}=0.51 ; 95 \% \mathrm{Cl}=0.28-0.93 ; \mathrm{p}=0.028)$, but not for $\mathrm{MM}$. A positive association was found between GSTP1 rs1695 AG + GG vs. AA genotypes for MM when compared to pleural plaques (OR = 1.39; $95 \%$ $\mathrm{Cl}=1.00-1.94 ; \mathrm{p}=0.049$ ). The interactions between different polymorphisms showed no significant influence on the risk of investigated asbestos-related diseases. The interaction between GSTI null polymorphism and asbestos exposure decreased the MM risk $(\mathrm{OR}=0.17 ; 95 \% \mathrm{Cl}=0.03-0.85 ; \mathrm{p}=0.031)$.

Conclusions. Our findings suggest that GSTI null genotype may be associated with a decreased risk for pleural plaques and asbestosis, may modify the association between asbestos exposure and $\mathrm{MM}$ and may consequently act protectively on MM risk. This study also revealed a protective effect of the interaction between GSTPI rs 1695 polymorphism and asbestos exposure on MM risk.
\end{abstract}

Key words: polymorphisms; glutathione-related genes; asbestos; asbestosis; pleural plaques; malignant mesothelioma

\section{Introduction}

Asbestos exposure, which still represents an important health problem worldwide, is known to be associated with the development of asbestosrelated diseases, including benign pleural diseases (e.g. pleural plaques), asbestosis, lung cancer, malignant mesothelioma (MM) and other types of cancer ${ }^{1,2}$ The pathogenesis of asbestos-related diseases is complicated and not entirely elucidated. Nevertheless, numerous studies have suggested that in addition to a direct mechanical injury, asbestos may stimulate the production of reactive oxygen and nitric species (ROS and RNS) that were shown to have an important role in the pathogenesis of these diseases. ROS and RNS may cause 
asbestos-related lung injury, DNA strand breaks in mesothelial cells and may increase the risk for developing malignancy. ${ }^{3-5}$

To detoxify ROS and consequently prevent the adverse effects of oxidative stress, the human organism possesses antioxidant defence systems. Glutathione (GSH), a tripeptide composed from glutamic acid, cysteine and glycine, is an abundant cellular antioxidant which has a major role in the protection against oxidative injury in cells. It serves as a substrate of many antioxidative enzymes. ${ }^{6,7}$ The antioxidant capacity of the glutathione system depends on enzymes involved in its biosynthesis, such as glutamate cysteine ligase (GCL), also known as gamma glutamylcysteine synthetase, as well as on detoxification enzymes, such as glutathione S-transferases (GSTs). ${ }^{6,8-10}$

GCL is the rate limiting enzyme of the GSH synthesis and it is suggested to be the major factor that determines GSH level in healthy subjects. The enzyme consists of two subunits: a heavy catalytic subunit (GCLC) and a light modifier subunit (GCLM). ${ }^{6,10}$ High GSH concentration levels found in many tumors have been associated with the increased GCL activity. ${ }^{11,12}$

GSTs are phase II detoxifying enzymes involved in the inactivation of the electrophiles produced by ROS and RNS by catalyzing the conjugation of electrophilic compounds with reduced glutathione. ${ }^{8,9}$ In mammals, seven classes of cytosolic GST isoenzymes have been recognized: Alpha, Mu, Pi, Sigma, Theta, Omega and Zeta. ${ }^{13}$ The crucial GST enzyme in the human lung, which belongs to the Pi class, is GSTP1. ${ }^{14,15}$ Two other important polymorphic GSTs are GSTM1 (Mu class) and GSTT1 (Theta class). ${ }^{15,16}$

Genes coding for GSH related enzymes are polymorphic. Among the most commonly investigated promoter polymorphisms of the GCLC and GCLM genes are GCLC rs17883901 (c.-129C >T) and GCLM rs41303970 (c.-590 C>T). ${ }^{17-20}$ Some studies indicated that polymorphisms in GCLC and GCLM genes are associated with low levels of reduced GSH in vitro, which may explain susceptibility to certain diseases related to oxidative stress. ${ }^{17,18}$ The GCLC rs17883901 polymorphism has been suggested to suppress the GCLC gene induction response to oxidants and it has been implicated in coronary endothelial dysfunction and myocardial infarction. ${ }^{17}$ GCLC rs17883901 has also been proposed to modulate the renal disease risk in type 1 diabetes patients. ${ }^{21}$ The presence of GCLC rs17883901 T allele and GCLM rs41303970 $\mathrm{T}$ allele has also been associated with an increased risk of ischemic heart disease. ${ }^{19}$ However, according to the available literature the association between GCLC and GCLM polymorphisms and asbestos-related diseases has not been studied so far.

Regarding GSTM1 and GSTT1 genes, the most common polymorphism is due to homozygous deletion of these genes (null genotype), which results in the lack of the GSTM1 and GSTT1 enzyme activity. ${ }^{22,23}$ In the GSTP1 gene, two common single nucleotide polymorphisms (SNPs) have been described that lead to amino acid substitution and consequently reduced enzyme conjugating activity: GSTP1 rs1138272 (p.Ala114Val) and GSTP1 rs1138272 (p.Ala114Val). ${ }^{22}$ Hirvonen et al. reported an increased risk for developing $\mathrm{MM}$ for individuals with GSTM1 null genotype. ${ }^{24}$ Similarly, Landi et al. found an increased risk for MM in subject with GSTM1 null allele, while no effect was observed for GSTP1 and GSTT1 polymorphisms. ${ }^{25}$ In the study of Kukkonen et al., GSTT1 null genotype increased the risk for asbestos-related severe fibrotic changes and GSTM1 null genotype was associated with the greatest thickness of the pleural plaques. ${ }^{26}$ Our former study showed that asbestosis was associated with GSTT1 null genotype, but not with GSTM1 null genotype. ${ }^{27}$ Furthermore, we have reported the influence of GSTP1 rs1695 on the asbestosis risk, while no association was found between GSTP1 rs1138272 and asbestosis risk. ${ }^{28}$

The present study aimed to investigate the influence of GCLC, GCLM, GSTM1, GSTT1 and GSTP1 polymorphisms on the risk for developing pleural plaques, asbestosis and MM. In addition, we also investigated the influence of gene-gene interactions and interactions between glutathione-related polymorphisms and asbestos exposure on the risk for developing these diseases.

\section{Subjects and methods}

\section{Study population}

The cross sectional study included all together 940 asbestos-exposed subjects, among them 390 subjects with pleural plaques, 147 subjects with asbestosis, 225 subjects with MM and 178 subjects with no asbestos-related disease. Subjects with pleural plaques, asbestosis and MM were considered as cases, and those with no asbestos-related disease as controls.

Additionally, comparison was made between subjects with $\mathrm{MM}$ and subjects with pleural plaques. 
Subjects with pleural plaques, asbestosis and subjects with no asbestos-related disease were presented at the State Board for the Recognition of Occupational Asbestos Diseases in the period from 1 January 1998 to 31 December 2007 and were all occupationally exposed to asbestos. The information on all the subjects included was revised in 2018 to verify the latest diagnoses of asbestosrelated diseases. Subjects with MM were recruited at the Institute of Oncology Ljubljana, where they were treated in the period between 1 February 2004 and 31 December 2018. The study was approved by the Slovenian Ethics Committee for Research in Medicine and was carried out according to the Declaration of Helsinki.

\section{Clinical diagnosis}

The diagnosis of pleural plaques, asbestosis or "no asbestos-related disease" was verified by two groups of experts of the State Board for the Recognition of Occupational Asbestos Diseases, each group consisting of a specialist of occupational medicine, a pulmonologist, and a radiologist. Subjects with pleural MM were diagnosed by ultrasound-guided biopsy or thoracoscopy and those with peritoneal MM by laparoscopy. The diagnosis of MM was proved histopathologically by a pathologist experienced in diagnosing this malignant disease.

\section{Smoking and asbestos exposure}

Data on smoking were collected during an interview based on a standardized questionnaire. The number of pack-years of smoking was calculated from the duration of smoking and the number of cigarettes smoked per day.

Data on cumulative asbestos exposure in fibres/ $\mathrm{cm}^{3}$-years were available for the subjects with pleural plaques, asbestosis, "no asbestos-related disease" and for 28 patients with MM. Based on the data on cumulative asbestos exposure, the asbestos exposures in these subjects were divided into three groups: low (<11 fibres/ $\mathrm{cm}^{3}$-years), medium (11-20 fibres $/ \mathrm{cm}^{3}$-years) and high ( $>20$ fibres $/ \mathrm{cm}^{3}$-years) asbestos exposure. For the subjects with MM who lacked the data on cumulative asbestos exposure, asbestos exposures were assessed based on the precise work history and comparison with exposures of the group of subjects with known cumulative asbestos exposure. Accordingly, their asbestos exposures were divided into three groups with presumed low, medium or high asbestos exposure.

\section{DNA extraction and genotyping}

Genomic DNA was extracted from peripheral blood samples using Qiagen FlexiGene Kit (Qiagen, Hilden, Germany) according to the manufacturer's instructions.

GSTP1 rs1695, GSTP1 rs1138272, GCLC rs17883901, and GCLM rs41303970 genotypes were determined using competitive allele-specific polymerase chain reaction (KASP) assays (LGC Genomics, UK) following the manufacturer's instructions. Homozygous GSTM1 and GSTT1 gene deletions (null genotype) were determined using multiplex PCR in a single reaction as previously described with $H B B$ gene serving as a positive control. ${ }^{29}$

\section{Statistical methods}

Standard descriptive statistics was used to describe central tendency and variability of investigated variables. Chi-square test and Kruskal-Wallis test were used to compare categorical and continuous variables among different groups, respectively. Deviation from Hardy-Weinberg equilibrium (HWE) was also evaluated using chi-square test. Dominant and additive genetic models were used in the analysis. To compare genotype frequencies among groups, univariable and multivariable logistic regression models were used and odds ratios (ORs) with 95\% confidence intervals (CIs) were calculated. Characteristics used for adjustment in multivariable analysis were selected using stepwise forward-conditional logistic regression. The possible interactions between genotypes as well as between genetic polymorphisms, and between genetic polymorphisms and asbestos exposure were tested by logistic regression models using dummy variables.

Statistical analysis was carried out with IBM SPSS Statistics version 21.0 (IBM Corporation, Armonk, NY, USA). All statistical tests were twosided and the level of significance was set at 0.05.

\section{Results}

The characteristics of the groups of subjects with pleural plaques, asbestosis, MM and subjects without asbestos-related disease are presented in Table 1. A statistically significant difference between the groups was observed for the age ( $p<$ $0.001)$, pack-years of smoking $(\mathrm{p}=0.024)$ and asbestos exposure $(p<0.001)$. The mean age was the highest for subjects with MM (65 \pm 10.7 years), followed by subjects with asbestosis ( $58.7 \pm 9.1$ years). 
TABLE 1. Characteristics of subjects without asbestos-related disease, subjects with pleural plaques, asbestosis or malignant mesothelioma

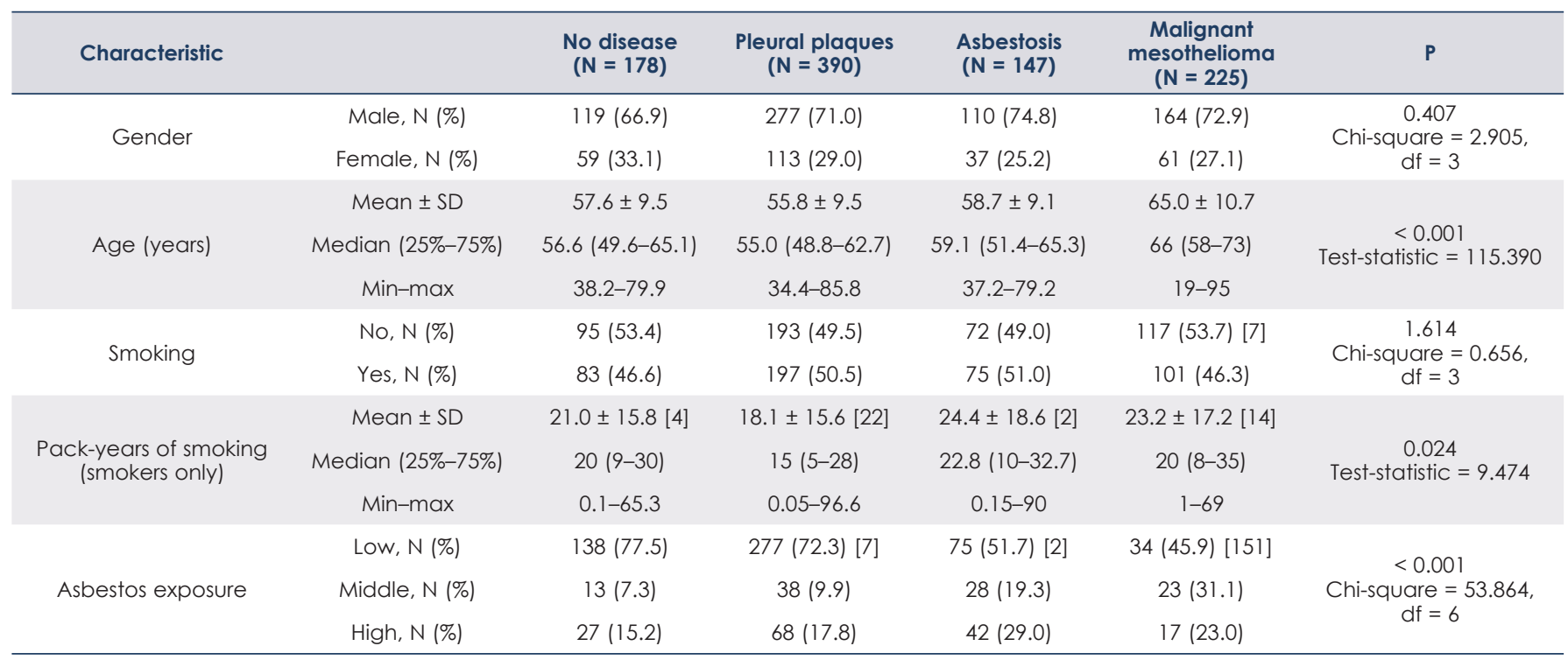

Number of missing data is presented in [] brackets. P-values were calculated using chi-square test for categorical or Kruskal-Wallis test for continuous variables. SD = standard deviation

The mean values of pack-years of smoking were the highest in subjects with asbestosis $(24.4 \pm 18.6)$ and in subjects with MM (23.2 \pm 17.2$)$. Regarding asbestos exposure, the percent of subjects with low asbestos exposure was the highest for the group of subject with no asbestos-related disease (77.5\%), followed by the group of subjects with pleural plaques $(72.3 \%)$ (Table 1$)$.

The genotype frequencies for all studied genetic polymorphisms are shown in Table 2. Genotype frequencies for all investigated SNPs were concordant with HWE.

TABLE 2. Genotype frequencies in all subjects, subjects without asbestos-related disease, subjects with pleural plaques, asbestosis and malignant mesothelioma

\begin{tabular}{|c|c|c|c|c|c|c|}
\hline Polymorphism & Genotype & $\begin{array}{l}\text { All subjects } \\
(\mathrm{N}=940)\end{array}$ & $\begin{array}{l}\text { No disease } \\
(\mathrm{N}=178)\end{array}$ & $\begin{array}{l}\text { Pleural plaques } \\
\qquad(\mathrm{N}=416)\end{array}$ & $\begin{array}{l}\text { Asbestosis } \\
(N=160)\end{array}$ & $\begin{array}{c}\text { Malignant } \\
\text { mesothelioma } \\
(\mathrm{N}=154)\end{array}$ \\
\hline \multirow{2}{*}{$\begin{array}{c}\text { GCLC rs } 17883901 \\
\text { C. }-129 C>T\end{array}$} & $\mathrm{CC}$ & $772(82.1)$ & 149 (83.7) & $310(79.5)$ & $124(84.4)$ & $189(84)$ \\
\hline & TT & $6(0.6)$ & $0(0)$ & $2(0.5)$ & $0(0)$ & $4(1.8)$ \\
\hline $\begin{array}{c}\text { GCLM rs } 41303970 \\
\text { C. }-590 \mathrm{C}>\mathrm{T}\end{array}$ & $\mathrm{CC}$ & $581(61.8)$ & $114(64)$ & $233(59.7)$ & $87(59.2)$ & $147(65.3)$ \\
\hline \multirow{2}{*}{ GSTMI Gene deletion } & present & $384(40.9)$ & $74(41.6)$ & $159(40.8)$ & $64(43.5)$ & 87 (38.7) \\
\hline & null genotype & $556(59.1)$ & $104(58.4)$ & $231(59.2)$ & $83(56.5)$ & $138(61.3)$ \\
\hline \multirow{2}{*}{ GSTII Gene deletion } & present & 782 (83.2) & $138(77.5)$ & $330(84.6)$ & $128(87.1)$ & $186(82.7)$ \\
\hline & null genotype & $158(16.8)$ & $40(22.5)$ & $60(15.4)$ & 19 (12.9) & 39 (17.3) \\
\hline \multirow{3}{*}{$\begin{array}{l}\text { GSTP1 rs } 1138272 \\
\text { p.Ala1 14Val }\end{array}$} & $\mathrm{CC}$ & 785 (83.5) & $141(79.2)$ & $334(85.6)$ & $121(82.3)$ & $189(84)$ \\
\hline & СT & $146(15.5)$ & $34(19.1)$ & $54(13.8)$ & $23(15.6)$ & $35(15.6)$ \\
\hline & $\mathrm{TT}$ & $9(1.0)$ & $3(1.7)$ & $2(0.5)$ & $3(2)$ & $1(0.4)$ \\
\hline
\end{tabular}


TABLE 3. The association between different asbestos-related diseases and genotypes in univariate analysis

\begin{tabular}{|c|c|c|c|c|c|c|c|c|c|c|c|}
\hline Polymorphism & Genotype & \multicolumn{2}{|c|}{$\begin{array}{l}\text { Asbestos-related disease } \\
\text { vs. no disease }\end{array}$} & \multicolumn{2}{|c|}{$\begin{array}{l}\text { Pleural plaques } \\
\text { vs. no disease }\end{array}$} & \multicolumn{2}{|c|}{$\begin{array}{c}\text { Asbestosis } \\
\text { vs. no disease }\end{array}$} & \multicolumn{2}{|c|}{$\begin{array}{c}\text { MM } \\
\text { vs. no disease }\end{array}$} & \multicolumn{2}{|c|}{$\begin{array}{c}\text { MM } \\
\text { vs. plaques }\end{array}$} \\
\hline \multirow{2}{*}{$\begin{array}{c}\mathrm{GCLC} \\
\text { rs } 17883901\end{array}$} & $\mathrm{CC}$ & Reference & & Reference & & Reference & & Reference & & Reference & \\
\hline & $\mathrm{CT}+\mathrm{TT}$ & $1.15(0.74-1.78)$ & 0.541 & $1.33(0.83-2.12)$ & 0.237 & $0.95(0.52-1.73)$ & 0.874 & $0.98(0.57-1.67)$ & 0.937 & $0.74(0.48-1.14)$ & 0.169 \\
\hline \multirow{3}{*}{$\begin{array}{l}\text { GCLM } \\
\text { rs } 41303970\end{array}$} & $\mathrm{CC}$ & Reference & & Reference & & Reference & & Reference & & Reference & \\
\hline & CT & $1.14(0.80-1.63)$ & 0.476 & $1.22(0.83-1.80)$ & 0.308 & $1.24(0.77-1.99)$ & 0.378 & $0.95(0.61-1.46)$ & 0.809 & $0.77(0.54-1.11)$ & 0.164 \\
\hline & $\mathrm{CT}+\mathrm{TT}$ & $1.13(0.80-1.58)$ & 0.495 & $1.20(0.83-1.73)$ & 0.330 & $1.23(0.78-1.93)$ & 0.369 & $0.95(0.63-1.43)$ & 0.788 & $0.79(0.56-1.11)$ & 0.170 \\
\hline \multirow[b]{2}{*}{ GSTMI } & present & Reference & & Reference & & Reference & & Reference & & Reference & \\
\hline & $\begin{array}{c}\text { null } \\
\text { genotype }\end{array}$ & $1.04(0.74-1.44)$ & 0.828 & $1.03(0.72-1.48)$ & 0.857 & $0.92(0.59-1.44)$ & 0.721 & $1.13(0.76-1.69)$ & 0.554 & $1.09(0.78-1.53)$ & 0.608 \\
\hline GSTII & present & Reference & & Reference & & Reference & & Reference & & Reference & \\
\hline \multirow{2}{*}{ GSTPI rs1695 } & GG & $0.80(0.45-1.40)$ & 0.428 & $0.67(0.36-1.25)$ & 0.208 & $0.86(0.41-1.80)$ & 0.698 & $1.01(0.51-1.97)$ & 0.988 & $1.50(0.84-2.67)$ & 0.170 \\
\hline & $A G+G G$ & $0.80(0.58-1.11)$ & 0.185 & $0.73(0.51-1.04)$ & 0.078 & $0.73(0.47-1.13)$ & 0.157 & $1.01(0.68-1.5)$ & 0.958 & $1.39(1.00-1.94)$ & 0.049 \\
\hline \multirow{2}{*}{$\begin{array}{c}\text { GSTPI } \\
\text { rS1 } 138272\end{array}$} & $\mathrm{CC}$ & Reference & & Reference & & Reference & & Reference & & Reference & \\
\hline & $\mathrm{CT}+\mathrm{TT}$ & $0.70(0.46-1.05)$ & 0.087 & $0.64(0.40-1.01)$ & 0.056 & $0.82(0.47-1.43)$ & 0.482 & $0.73(0.44-1.21)$ & 0.216 & $1.14(0.72-1.79)$ & 0.583 \\
\hline
\end{tabular}

Statistically significant results are printed in bold. $M M=$ malignant mesothelioma

In univariate logistic regression analysis, no association was found between GCLC rs17883901 and GCLM rs41303970 genetic polymorphisms and asbestos-related diseases.

GSTT1 null genotype was associated with the decreased risk for asbestos-related diseases when analysed together $(\mathrm{OR}=0.63 ; 95 \% \mathrm{CI}=0.42-0.95 ; \mathrm{p}$ $=0.026$ ). When analysing the risk for each disease separately, GSTT1 null genotype was associated with the decreased risk for pleural plaques (OR $=0.63 ; 95 \% \mathrm{CI}=0.40-0.98 ; \mathrm{p}=0.026)$ and asbestosis $(\mathrm{OR}=0.51 ; 95 \% \mathrm{CI}=0.28-0.93 ; \mathrm{p}=0.028)$, but not for MM. No association was found between GSTM1 null genotype and asbestos-related diseases. Regarding GSTP1 polymorphisms, a positive association was found between GSTP1 rs1695 AG + GG vs. AA genotypes for MM only when compared to pleural plaques $(\mathrm{OR}=1.39 ; 95 \% \mathrm{CI}=$ 1.00-1.94; $\mathrm{p}=0.049$ ) (Table 3).

Regarding age, no association was found between age and pleural plaques (OR $=0.98 ; 95 \% \mathrm{CI}$ $=0.96-1.00 ; \mathrm{p}=0.032)$. A slight association was observed between age and $\mathrm{MM}(\mathrm{OR}=1.07 ; 95 \% \mathrm{CI}=$ 1.05-1.10; $\mathrm{p}<0.001)$, as well as between age and $\mathrm{MM}$ when compared to pleural plaques $(\mathrm{OR}=1.10$; $95 \% \mathrm{CI}=1.08-1.12 ; \mathrm{p}<0.001$ ).
The analysis of association between asbestos exposure and asbestos-related diseases revealed a positive association between high and medium vs. low asbestos exposure and all asbestos-related diseases $(\mathrm{OR}=1.93 ; 95 \% \mathrm{CI}=1.31-2.85 ; \mathrm{p}=0.001)$, between high and medium vs. low asbestos exposure and asbestosis $(\mathrm{OR}=3.22 ; 95 \% \mathrm{CI}=1.99-5.20$; $\mathrm{p}<0.001$ ), and between high and medium vs. low asbestos exposure and $\mathrm{MM}(\mathrm{OR}=4.06 ; 95 \% \mathrm{CI}=$ 2.28-7.23; $\mathrm{p}<0.001)$. When analysing the association between high and medium vs. low asbestos exposure and MM compared to pleural plaques, the OR was 3.07 (95\% CI = 1.85-5.12; p < 0.001).

In multivariate logistic regression analysis, the risk of GSTT1 null genotype for all asbestosrelated diseases together $(\mathrm{OR}=0.62 ; 95 \% \mathrm{CI}=$ $0.41-0.94 ; \mathrm{p}=0.025)$ and separately for asbestosis $(\mathrm{OR}=0.51 ; 95 \% \mathrm{CI}=0.27-0.95 ; \mathrm{p}=0.033) \mathrm{did}$ not change considerably after adjustment for asbestos exposure. Similarly, the risk of GSTT1 null genotype for pleural plaques remained practically unchanged after adjustment for age (OR $=0.63 ; 95 \%$ $\mathrm{CI}=0.40-0.99 ; \mathrm{p}=0.046)$. On the contrary, the risk of GSTP1 rs1695 AG + GG vs. AA genotypes for MM compared to pleural plaques increased slightly $(\mathrm{OR}=1.97 ; 95 \% \mathrm{CI}=1.14-3.39 ; \mathrm{p}=0.015)$ 
TABLE 4. The association between different asbestos-related diseases and genotypes in multivariate analysis

\begin{tabular}{|c|c|c|c|c|c|c|c|c|c|c|c|}
\hline Polymorphism & Genotype & \multicolumn{2}{|c|}{$\begin{array}{l}\text { Asbestos-related disease } \\
\text { vs. no disease }\end{array}$} & \multicolumn{2}{|c|}{$\begin{array}{l}\text { Pleural plaques } \\
\text { vs. no disease }\end{array}$} & \multicolumn{2}{|c|}{$\begin{array}{c}\text { Asbestosis } \\
\text { vs. no disease }\end{array}$} & $\begin{array}{c}\text { MM } \\
\text { vs. no disease } \\
\text { OR }(95 \% \mathrm{Cl})\end{array}$ & $P$ & \multicolumn{2}{|c|}{$\begin{array}{c}\text { MM } \\
\text { vs. plaques }\end{array}$} \\
\hline $\begin{array}{c}\text { GCLC } \\
\text { rs17883901 }\end{array}$ & $\mathrm{CC}$ & Reference & & Reference & & Reference & & Reference & & Reference & \\
\hline \multirow{3}{*}{$\begin{array}{c}\text { GCLM } \\
\text { rs } 41303970\end{array}$} & $\mathrm{CC}$ & Reference & & Reference & & Reference & & Reference & & Reference & \\
\hline & CT & $1.16(0.8-1.68)$ & 0.431 & $1.22(0.82-1.79)$ & 0.323 & $1.10(0.67-1.81)$ & 0.695 & $0.98(0.51-1.87)$ & 0.945 & $0.76(0.43-1.36)$ & 0.360 \\
\hline & $\mathrm{CT}+\mathrm{TT}$ & $1.16(0.82-1.64)$ & 0.406 & $1.19(0.82-1.72)$ & 0.351 & $1.14(0.72-1.83)$ & 0.576 & $1.00(0.55-1.84)$ & 0.994 & $0.80(0.47-1.38)$ & 0.429 \\
\hline \multirow[b]{2}{*}{ GSTMI } & present & Reference & & Reference & & Reference & & Reference & & Reference & \\
\hline & $\begin{array}{c}\text { null } \\
\text { genotype }\end{array}$ & $1.04(0.74-1.46)$ & 0.837 & $1.06(0.74-1.53)$ & 0.738 & $0.84(0.53-1.34)$ & 0.464 & $1.09(0.60-1.98)$ & 0.774 & $1.09(0.63-1.87)$ & 0.756 \\
\hline GSTI & present & Reference & & Reference & & Reference & & Reference & & Reference & \\
\hline \multirow{2}{*}{ GSTPI rs1695 } & GG & $0.8(0.45-1.43)$ & 0.461 & $0.67(0.36-1.24)$ & 0.203 & $0.96(0.45-2.06)$ & 0.920 & $1.65(0.65-4.16)$ & 0.288 & $2.40(1.04-5.54)$ & 0.039 \\
\hline & $A G+G G$ & $0.78(0.56-1.1)$ & 0.153 & $0.72(0.51-1.04)$ & 0.077 & $0.71(0.45-1.12)$ & 0.140 & $1.31(0.72-2.39)$ & 0.370 & $1.97(1.14-3.39)$ & 0.015 \\
\hline \multirow{2}{*}{$\begin{array}{l}\text { GSTP1 } \\
\text { rs } 1138272\end{array}$} & $\mathrm{CC}$ & Reference & & Reference & & Reference & & Reference & & Reference & \\
\hline & $\mathrm{CT}+\mathrm{TT}$ & $0.68(0.44-1.04)$ & 0.078 & $0.64(0.4-1.02)$ & 0.059 & $0.84(0.47-1.51)$ & 0.565 & $0.73(0.34-1.60)$ & 0.433 & $1.02(0.49-2.13)$ & 0.965 \\
\hline
\end{tabular}

$M M=$ malignant mesothelioma. Statistically significant results are printed in bold

Adjustments made: Asbestos-related disease vs. no disease, Asbestosis vs. no disease: adjusted for asbestos exposure; Pleural plaques vs. no disease: adjusted for age; MM vs. no disease, MM vs. plaques: adjusted for asbestos exposure, age

after adjustment for asbestos exposure and age (Table 4).

In further logistic regression analysis, the interactions between polymorphisms showed no significant influence on the risk for developing asbestosrelated diseases (data not shown).

Testing the influence of interactions between asbestos high and medium vs. low exposure and genetic polymorphisms on the risk of asbestos-related diseases, the interaction between asbestos exposure and GSTT1 null polymorphism decreased the risk for developing $\mathrm{MM}(\mathrm{OR}=0.17 ; 95 \% \mathrm{CI}=$ $0.03-0.85 ; \mathrm{p}=0.031)$. Similarly, the interaction between asbestos exposure and GSTT1 null polymorphism $(\mathrm{OR}=0.11 ; 95 \% \mathrm{CI}=0.02-0.49 ; \mathrm{p}=0.004)$ and the interaction between asbestos exposure and GSTP1 rs1695 polymorphism (OR $=0.14 ; 95 \% \mathrm{CI}$ $=0.03-0.65 ; \mathrm{p}=0.012$ ) decreased the risk of $\mathrm{MM}$ when compared to pleural plaques.

\section{Discussion}

The present study investigated the influence of genetic polymorphisms in GSH related genes, the interactions between these polymorphism, and interactions between polymorphisms and asbestos exposure on the risk of asbestos-related diseases.

The present study revealed a protective effect of GSTT1 null genotype on the risk of all studied asbestos-related diseases together and particularly on the risk of pleural plaques and asbestosis. The explanation of these findings could be that in some instances GSTT1 may catalyse toxification and not detoxification reaction, leading to even more reactive conjugate. ${ }^{15}$ This observation is in agreement with the results of our previous study, in which GSTT1 null genotype also decreased the asbestosis risk. ${ }^{27}$ On the other hand, in the present study GSTM1 null genotype showed no effect on the risk of asbestos-related diseases, which is also consistent with the results of our previous study. ${ }^{27}$ Similar findings were observed by Jakobsson et al., who reported no association between GSTM1 deficiency and parenchymal and pleural abnormalities among the workers exposed to asbestos ${ }^{30}$, and also by Hirvonen et al., who revealed no increased risk for the asbestos-related pulmonary disorders in subjects with homozygous deletion of GSTM1 gene. ${ }^{16}$ Contrary to the results of our study, 
Kukkonen et al. reported that GSTT1 null genotype increased the risk of asbestosis and GSTM1 null genotype was related to the greatest thickness of the pleural plaques. ${ }^{26}$ Although Landi et al. observed an increased risk for MM in subjects bearing GSTM1 null allele ${ }^{25}$, in our current study, no association was found between either GSTM1 null genotype or GSTT1 null genotype and MM risk.

The results of our study showed that GSTP1 rs1695 AG + GG vs. AA genotypes increased the MM risk, while GSTP1 rs1138272 polymorphism did not affect the risk of this malignoma. On the contrary, GSTP1 polymorphisms did not influence the $\mathrm{MM}$ risk in the study by Landi et al. ${ }^{25}$

Our study revealed no influence of GCLC rs17883901 and GCLM rs41303970 on the risk for asbestos-related diseases. Our results suggest that these two polymorphisms are not related to the susceptibility to asbestos-related diseases. To our knowledge, no other studies investigated the role of polymorphic genes involved in GSH synthesis in asbestos-related diseases.

Our study confirmed the impact of high and medium vs. low asbestos exposure on the risk for asbestosis and MM, which is consistent with the findings of previous studies. ${ }^{31-34}$ However, our results also showed that nearly $46 \%$ of subjects with MM, $52 \%$ of subjects with asbestosis and $72 \%$ subjects with pleural plaques had low asbestos exposure. This suggests that asbestos-related diseases can also develop when asbestos exposures are low, which was indicated especially for MM. ${ }^{35,36}$

In this study, the interactions between investigated GSH related gene polymorphisms did not influence the risk for developing asbestos-related diseases. On the other hand, we observed that the interaction between GSTT1 null polymorphism and asbestos exposure decreased the risk for developing MM, although there was no independent association between GSTT1 null and MM when compared to controls with no asbestos-related disease. In other words, GSTT1 null genotype modified the association between high and medium vs. low asbestos exposure and MM and acted protectively on the risk of this malignant disease.

Another interesting finding of this study showed that the interaction between GSTP1 rs1695 AG + GG vs. AA genotypes and asbestos exposure decreased the risk of MM when compared to pleural plaques, despite the fact that in univariate analysis both GSTP1 polymorphism and asbestos exposure were associated with an increased risk of MM. The relation between benign pleural plaques and the risk of MM has not been clearly proved so far.
Although pleural plaques may be the endpoint and the development of pleural plaques may be an entirely independent process from the development of $\mathrm{MM}^{37}$, it is likely that there is a relation between pleural plaques and MM. ${ }^{38}$ The present study suggests a modifying and protective effect of GSTP1 rs1695 genotypes on the association between asbestos exposure and MM risk when compared to pleural plaques.

Considering the potential limitations of the study, the data on asbestos exposure were not available for all subjects, especially not for patients with MM. Consequently, the analyses of the interactions between genetic polymorphisms and asbestos exposure could be performed only for the subgroup of MM patients.

On the other hand, the study also brings novel findings and has some important strengths. Firstly, according to our knowledge, this is the first study to investigate the association between GCLC rs17883901 and GCLM rs41303970 genetic polymorphisms and asbestos-related diseases. Secondly, it included relatively large numbers of subjects with different asbestos-related diseases from genetically homogenous population and investigated functional genetic polymorphisms in different GSH related genes.

In conclusion, our findings suggest that among genetic polymorphisms in GSH related genes, GSTT1 null polymorphism may be associated with the risk for developing pleural plaques and asbestosis and may also modify the association between asbestos exposure and MM and therefore act protectively on the risk for this malignoma. This study also revealed a modifying and protective effect of GSTP1 rs1695 polymorphism on the association between asbestos exposure and MM risk when pleural plaques were considered as controls.

\section{Acknowledgements and funding}

The authors would like to thank Savica Soldat, Urška Slapšak, Ana Cirnski and Maj Bavec for their help with genotyping analyses. This work was financially supported by the Slovenian Research Agency (ARRS Grants Nos. P1-0170, L3-8203 and L3-2622).

\section{References}

1. Stayner L, Welch LS, Lemen R. The worldwide pandemic of asbestos-related diseases. Annu Rev Public Health 2013; 34: 205-16. doi: 10.1146/annurevpublhealth-031811-124704 
2. Vainio H. Epidemics of asbestos-related diseases - something old, something new. Scand J Work Environ Health 2015; 41: 1-4. doi: 10.5271/ sjweh.3471

3. Kamp DW, Graceffa P, Pryor WA, Weitzman SA. The role of free radicals in asbestos-induced diseases. Free Radic Biol Med 1992; 12: 293-315. doi: 10.1016/0891-5849(92)90117-y

4. Kinnula VL. Oxidant and antioxidant mechanisms of lung disease caused by asbestos fibres. Eur Respir J 1999; 14: 706-16. doi: 10.1034/j.13993003.1999.14c35.x

5. Solbes E, Harper RW. Biological responses to asbestos inhalation and pathogenesis of asbestos-related benign and malignant disease. J Investig Med 2018; 66: 721-7. doi: 10.1136/jim-2017-000628

6. Nichenametla SN, Muscat JE, Liao JG, Lazarus P, Richie JP, Jr. A functional trinucleotide repeat polymorphism in the $5^{\prime}$-untranslated region of the glutathione biosynthetic gene GCLC is associated with increased risk for lung and aerodigestive tract cancers. Mol Carcinog 2013; 52: 791-9. doi: 10.1002/mc. 21923

7. Zhang $\mathrm{H}$, Liu H, Zhou L, Yuen J, Forman HJ. Temporal changes in glutathione biosynthesis during the lipopolysaccharide-induced inflammatory response of THP-1 macrophages. Free Radic Biol Med 2017; 113: 304-10. doi: 10.1016/j.freeradbiomed.2017.10.010

8. Ketterer B. A bird's eye view of the glutathione transferase field. Chem Biol Interact 2001; 138: 27-42. doi: 10.1016/s0009-2797(01)00277-0

9. Strange RC, Spiteri MA, Ramachandran S, Fryer AA. Glutathione-S transferase family of enzymes. Mutat Res 2001; 482: 21-6. doi: 10.1016/ s0027-5107(01)00206-8

10. Chen Y, Shertzer HG, Schneider SN, Nebert DW, Dalton TP. Glutamate cysteine ligase catalysis: dependence on ATP and modifier subunit for regulation of tissue glutathione levels. J Biol Chem 2005; 280: 33766-74. doi: 10.1074/jbc.M504604200

11. Traverso N, Ricciarelli R, Nitti M, Marengo B, Furfaro AL, Pronzato MA, et al. Role of glutathione in cancer progression and chemoresistance. Oxid Med Cell Longev 2013; 2013: 972913. doi: 10.1155/2013/972913

12. Zmorzynski S, Swiderska-Kolacz G, Koczkodaj D, Filip AA. Significance of polymorphisms and expression of enzyme-encoding genes related to glutathione in hematopoietic cancers and solid tumors. Biomed Res Int 2015 2015: 853573. doi: 10.1155/2015/853573

13. Flanagan JU, Smythe ML. Sigma-class glutathione transferases. Drug Metab Rev 2011; 43: 194-214. doi: 10.3109/03602532.2011.560157

14. Tan XL, Moslehi R, Han W, Spivack SD. Haplotype-tagging single nucleotide polymorphisms in the GSTP1 gene promoter and susceptibility to lung cancer. Cancer Detect Prev 2009; 32: 403-15. doi: 10.1016/j.cdp.2009.02.004

15. Hayes JD, Strange RC. Glutathione S-transferase polymorphisms and their biological consequences. Pharmacology 2000; 61: 154-66. doi: $10.1159 / 000028396$

16. Hirvonen A, Saarikoski ST, Linnainmaa K, Koskinen K, Husgafvel-Pursiainen $\mathrm{K}$, Mattson K, et al. Glutathione S-transferase and $\mathrm{N}$-acetyltransferase genotypes and asbestos-associated pulmonary disorders. J Natl Cancer Inst 1996; 88: 1853-6. doi: 10.1093/jnci/88.24.1853

17. Koide S, Kugiyama K, Sugiyama S, Nakamura S, Fukushima H, Honda O, et al. Association of polymorphism in glutamate-cysteine ligase catalytic subunit gene with coronary vasomotor dysfunction and myocardial infarction. J Am Coll Cardiol 2003; 41: 539-45. doi: 10.1016/s0735-1097(02)02866-8

18. Yuniastuti A, Susanti R, Mustikaningtyas D. Polymorphism of glutamatecysteine ligase subunit catalytic (GCLC) gene in pulmonary tuberculosis patients. Pak J Biol Sci 2017; 20: 397-402. doi: 10.3923/pjbs.2017.397.402

19. Skvortsova L, Perfelyeva A, Khussainova E, Mansharipova A, Forman $H$ Djansugurova L. Association of GCLM -588C/T and GCLC -129T/C promoter polymorphisms of genes coding the subunits of glutamate cysteine ligase with ischemic heart disease development in Kazakhstan population. Dis Markers 2017; 2017: 4209257. doi: 10.1155/2017/4209257

20. Li J, Yin F, Lin Y, Gao M, Wang L, Liu S, et al. Genetic susceptibility analysis of GCLC rs17883901 polymorphism to preeclampsia in Chinese Han women. Gynecol Endocrinol 2020; 36: 781-5. doi: 10.1080/09513590.2020.1725970
21. Vieira SM, Monteiro MB, Marques $T$, Luna AM, Fortes MA, Nery $M$, et al. Association of genetic variants in the promoter region of genes encoding p22phox (CYBA) and glutamate cysteine ligase catalytic subunit (GCLC) and renal disease in patients with type 1 diabetes mellitus. BMC Med Genet 2011; 12: 129. doi: 10.1186/1471-2350-12-129

22. Hayes JD, Flanagan JU, Jowsey IR. Glutathione transferases. Annu Rev Pharmacol Toxicol 2005; 45: 51-88. doi: 10.1146/annurev.pharmtox.45.120403.095857

23. Ali-Osman F, Akande O, Antoun G, Mao JX, Buolamwini J. Molecular cloning, characterization, and expression in Escherichia coli of full-length cDNAs of three human glutathione S-transferase Pi gene variants. Evidence for differential catalytic activity of the encoded proteins. J Biol Chem 1997; 272: 10004-12. doi: 10.1074/jbc.272.15.10004

24. Hirvonen A, Pelin K, Tammilehto L, Karjalainen A, Mattson K, Linnainmaa K. Inherited GSTM1 and NAT2 defects as concurrent risk modifiers in asbestosrelated human malignant mesothelioma. Cancer Res 1995; 55: 2981-3. PMID: 7606714

25. Landi S, Gemignani F, Neri M, Barale R, Bonassi S, Bottari F, et al. Polymorphisms of glutathione-S-transferase $\mathrm{M} 1$ and manganese superoxide dismutase are associated with the risk of malignant pleural mesothelioma. Int J Cancer 2007; 120: 2739-43. doi: 10.1002/ijc.22590

26. Kukkonen MK, Hamalainen S, Kaleva S, Vehmas T, Huuskonen MS, Oksa P, et al. Genetic susceptibility to asbestos-related fibrotic pleuropulmonary changes. Eur Respir J 2011; 38: 672-8. doi: 10.1183/09031936.00049810

27. Franko A, Dodic-Fikfak M, Arneric N, Dolzan V. Glutathione S-transferases GSTM1 and GSTT1 polymorphisms and asbestosis. J Occup Environ Med 2007; 49: 667-71. doi: 10.1097/JOM.0b013e318065b855

28. Franko A, Dolzan V, Arneric N, Dodic-Fikfak M. The influence of genetic polymorphisms of GSTP1 on the development of asbestosis. J Occup Environ Med 2008; 50: 7-12. doi: 10.1097/JOM.0b013e31815cbab5

29. Chen $\mathrm{CL}$, Liu Q, Relling MV. Simultaneous characterization of glutathione $\mathrm{S}$-transferase M1 and T1 polymorphisms by polymerase chain reaction in American whites and blacks. Pharmacogenetics 1996; 6: 187-91. doi: 10.1097/00008571-199604000-00005

30. Jakobsson K, Rannug A, Alexandrie AK, Rylander L, Albin M, Hagmar L. Genetic polymorphism for glutathione-S-transferase mu in asbestos cement workers. Occup Environ Med 1994; 51: 812-6. doi: 10.1136/oem.51.12 812

31. Jamrozik E, de Klerk N, Musk AW. Asbestos-related disease. Intern Med 2011; 41: 372-80. doi: 10.1111/j.1445-5994.2011.02451.x

32. Frank AL, Joshi TK. The global spread of asbestos. Ann Glob Health 2014; 80 : 257-62. doi: 10.1016/j.aogh.2014.09.016

33. Lacourt A, Lévêque E, Guichard E, Gilg Soit Ilg A, Sylvestre MP, Leffondré K. Dose-time-response association between occupational asbestos exposure and pleural mesothelioma. Occup Environ Med 2017; 74: 691-7. doi: 10.1136/oemed-2016-104133

34. Ulvestad B, Kjærheim K, Martinsen JI, Damberg G, Wannag A, Mowe G, et al. Cancer incidence among workers in the asbestos-cement producing industry in Norway. Scand J Work Environ Health 2002; 28: 411-7. doi: 10.5271/sjweh.693

35. Nishimura $\mathrm{Y}$, Kumagai-Takei $\mathrm{N}$, Matsuzaki $\mathrm{H}$, Lee $\mathrm{S}$, Maeda $\mathrm{M}$, Kishimoto $\mathrm{T}$, et al. Functional alteration of natural killer cells and cytotoxic T lymphocytes upon asbestos exposure and in malignant mesothelioma patients. Biomed Res Int 2015; 2015: 238431. doi: 10.1155/2015/238431

36. Rosner D, Markowitz G, Chowkwanyun M. "Nondetected": the politics of measurement of asbestos in talc, 1971-1976. Am J Public Health 2019; 109: 969-74. doi: 10.2105/ajph.2019.305085

37. Pairon JC, Laurent F, Rinaldo M, Clin B, Andujar P, Ameille J, et al. Pleura plaques and the risk of pleural mesothelioma. J Nat/ Cancer Inst 2013; 105: 293-301. doi: 10.1093/jnci/djs513

38. Maxim LD, Niebo R, Utell MJ. Are pleural plaques an appropriate endpoint for risk analyses? Inhal Toxicol 2015; 27: 321-34. doi: 10.3109/08958378.2015.1051640 